\title{
Zero Temperature Phases of the Electron Gas
}

\author{
G. Ortiz, ${ }^{1}$ M. Harris, ${ }^{2}$ and P. Ballone ${ }^{3,4}$ \\ ${ }^{1}$ Theoretical Division, Los Alamos National Laboratory, P.O. Box 1663, Los Alamos, New Mexico 87545 \\ ${ }^{2}$ Max-Planck Institut für Festkörperforschung, Heisenbergstrasse 1, 70569 Stuttgart, Germany \\ ${ }^{3}$ Institut für Festkörperforschung Forschungszentrum Jülich, 52425 Jülich, Germany \\ ${ }^{4}$ Istituto Nazionale per la Fisica della Materia, Universitá degli Studi di Messina, \\ Contrada Papardo, C.P. 50, 98166 Messina, Italy
}

(Received 16 September 1998)

\begin{abstract}
The stability of different phases of the three-dimensional nonrelativistic electron gas is analyzed using stochastic methods. With decreasing density, we observe a continuous transition from the paramagnetic to the ferromagnetic fluid, with an intermediate stability range $\left(20 \pm 5 \leq r_{s} \leq 40 \pm 5\right)$ for the partially spin-polarized liquid. The freezing transition into a ferromagnetic Wigner crystal occurs at $r_{s}=65 \pm 10$. We discuss the relative stability of different magnetic structures in the solid phase. [S0031-9007(99)09514-9]
\end{abstract}

PACS numbers: 71.10.Ca, 05.30.Fk, 75.10.Lp

Ever since the pioneering work of Wigner and Seitz [1] on the cohesive energy of metals, the calculation of the ground state energy of the interacting electron gas became the object of considerable theoretical interest [2]. Indeed, the electron gas provides the simplest model in which nontrivial magnetic structures and electron localization can be realized by varying a single parameter, namely, the average electron density $\rho$.

In the present paper we investigate the relative stability of various broken symmetry phases of the nonrelativistic three-dimensional electron gas, both fluid and solid, using stochastic methods. We find that the paramagnetic to ferromagnetic (full spin polarization) transition is not first order, but a continuous one, involving partial spin polarization states (weak ferromagnetism) [3]. Moreover we find that the transition to a Wigner crystal occurs at a significantly larger density than the value commonly accepted $[3,4]$ and that near the quantum freezing transition the fcc and bcc crystal phases are nearly degenerate.

The jellium model consists of $N$ electrons enclosed in a box of volume $\Omega$ (periodically repeated in space) in the presence of a neutralizing background of positive charge. Two parameters characterize its zero temperature phase diagram, namely, the particle density $\rho=N / \Omega$ and the spin polarization $\zeta=\left|N_{\uparrow}-N_{\downarrow}\right| / N$, where $N_{\uparrow(\downarrow)}$ is the number of spin-up(down) electrons $\left(N=N_{\uparrow}+N_{\downarrow}\right)$. The system is governed by the Hamiltonian (Hartree a.u.)

$$
\hat{H}=\sum_{i=1}^{N} \frac{\mathbf{p}_{i}^{2}}{2}+\sum_{i<j}^{N} \frac{1}{\left|\mathbf{r}_{i}-\mathbf{r}_{j}\right|}+\Lambda,
$$

where $\mathbf{r}_{i}$ and $\mathbf{p}_{i}$ are the position and linear momentum of particle $i$, and $\Lambda$ is a constant representing the effect of the background. Since we are interested in the macroscopic properties of this model system, the thermodynamic limit ( $N, \Omega \rightarrow \infty$, keeping $\rho$ constant) is to be performed in the end by finite size extrapolation.

Elementary scaling arguments indicate that the kinetic energy term in (1) goes as $1 / r_{s}^{2}\left(r_{s}\right.$ is the Wigner-Seitz radius in units of the Bohr radius and its relation to the density is $\rho^{-1}=\frac{4 \pi}{3} r_{s}^{3}$ ), while the potential energy scales as $1 / r_{s}$. Depending on the relative strength between Coulomb and kinetic energies, we can characterize three different regimes: the weak $\left(r_{s} \lesssim 1\right)$, intermediate $\left(1 \lesssim r_{s} \lesssim 10\right)$, and strong $\left(r_{s} \gtrsim 10\right)$ Coulomb coupling regimes. The random-phase approximation [2] provides an accurate description of the weak-coupling regime. The intermediate coupling region, of direct interest for density functional calculations, has been extensively studied by numerical [4,5] and semianalytic methods [6]. Not surprisingly, the least known regime is the strongly correlated one, for which an early quantum Monte Carlo calculation [4] is still the most authoritative study.

To delve into the strong coupling regime we employ the variational (VMC) and diffusion (DMC) quantum Monte Carlo methods. The starting point is provided by a variational wave function of the Jastrow type:

$\Psi_{T}(\mathcal{R})=J[\mathcal{R}, \Sigma] \operatorname{det}_{\uparrow}[\varphi] \cdot \operatorname{det}_{\downarrow}[\varphi], \quad \Psi_{T}(\mathcal{R}) \in \mathbb{R}$, where $\operatorname{det}_{\uparrow(\downarrow)}[\varphi]$ is a spin-up(down) Slater determinant of one-electron orbitals $\varphi$ that are either plane waves (fluid phases) or localized functions (crystal phases). In the equation above, $\mathcal{R}=\left(\mathbf{r}_{1}, \ldots, \mathbf{r}_{N}\right)$ and $\Sigma=\left(\sigma_{1}, \ldots, \sigma_{N}\right)$ represent the full set of positions and spins of the electrons in the system. Considering only 2-body correlations, the Jastrow factor can be written as $J[\mathcal{R}, \Sigma]=$ $\prod_{i<j}^{N} \exp \left[v_{i j}\left(\left|\mathbf{r}_{i}-\mathbf{r}_{j}\right|\right)\right]$. For an infinite system the optimal $v_{i j}(r)$ should decay as $1 / r$ at large distances to account for the correct plasmon dispersion relation. For large but finite systems, however, that is not the case and to reproduce the long distance behavior without resorting to expensive evaluations of $v$ 's by Ewald sums, we consider a finite range $v_{i j}$, vanishing outside a sphere of radius $R_{t}$ tangential to the unit cell: $v_{i j}(r)=0$ if $r>R_{t}$. We have verified that this truncation involves an insignificant loss of correlation energy at the variational level [7]. Inside the sphere $v_{i j}$, which is different for like and unlike spin electrons, is given by

$$
v_{i j}(r)=\bar{v}_{i j}(r)+A_{i j} \exp \left[-\alpha_{i j} r^{2}\right]
$$


and

$$
\bar{v}_{i j}= \begin{cases}\frac{a_{i j} r+b_{i j} r^{2}}{1+c_{i j} r+d_{i j} r^{2}}+s_{i j}, & r<R_{b}, \\ \sum_{k=0}^{5} f_{k}\left(r-R_{b}\right)^{k}, & r>R_{b} .\end{cases}
$$

The $\left\{a_{i j}\right\}$ are determined by the electron-electron cusp condition [8], while $\left\{b_{i j}, c_{i j}, d_{i j}, s_{i j}, A_{i j}, \alpha_{i j}\right\}$ and $R_{b}$ are variational parameters. The $\left\{f_{k}\right\}$ are chosen in such a way that $\Psi_{T}$ and its two first derivatives are continuous everywhere. The one-electron orbitals used for the crystal phases are exponentials of a Padé function:

$$
\varphi_{\mathbf{j}}(\mathbf{r})=\exp \left[\frac{-k_{1}\left|\mathbf{r}-\mathbf{R}_{\mathbf{j}}\right|^{2}}{1+k_{2}\left|\mathbf{r}-\mathbf{R}_{\mathbf{j}}\right|}\right],
$$

where the (positive) constants $k_{1}, k_{2}$ are also variational parameters, and the fixed vectors $\left\{\mathbf{R}_{\mathbf{j}}, \mathbf{j}=1, N\right\}$ are distributed on a regular (bcc or fcc) lattice. The fluid states are eigenstates of zero total momentum, while the solid ones are eigenstates of finite lattice translations with vanishing total crystal momentum. Both are eigenstates of the $z$ component of the total spin $\hat{S}_{z}$ with eigenvalue $\hbar M=\hbar\left(N_{\uparrow}-N_{\downarrow}\right) / 2$. Although the determinantal part of the wave function is also an eigenstate of $\hat{S}^{2}$, the Jastrow part may introduce some spin contamination. By analogy to what is found for other systems [9], we expect this effect to be small for carefully optimized wave functions. All the free coefficients are optimized by the variance minimization technique introduced in Ref. [10]. These wave functions are used to drive the DMC simulation [11].

Several features of the phase diagram discussed below depend upon the accurate evaluation of tiny energy differences. To provide a basis to estimate the reliability of our results, we report here the relevant aspects of our computation, including an estimate for the statistical and systematic errors. First of all, because of the high quality of the wave functions, and the relatively long runs, statistical errors are the least important source of uncertainties: for all $r_{s}$, the statistical error is less than $1 \%$ of the correlation energy. Moreover, the energy gain in going from $\mathrm{VMC}$ to DMC is relatively small. As expected, the difference $\delta E=E_{\mathrm{tot}}^{\mathrm{VMC}}-E_{\mathrm{tot}}^{\mathrm{DMC}}$ is largest at low $r_{s}$ [12], because the $n$-body contributions not included in our Jastrow function become more important at high density [13]. Moreover, for each $r_{s}$ we find that $\delta E(\zeta)$ is larger for $\zeta=0$ than for $\zeta=1$, and, in between, the $\zeta$ dependence of $\delta E$ is well represented by the simple interpolation: $\delta E(\zeta)=\delta E(0)+[\delta E(1)-\delta E(0)] \zeta^{2}$.

For the fluid phase the most important source of uncertainty in our calculations is the finite-size extrapolation to the thermodynamic limit. We applied the extrapolation scheme proposed in Ref. [4]:

$E_{\infty}(\boldsymbol{\zeta})=E_{N}(\boldsymbol{\zeta})-\Delta t_{N}(\boldsymbol{\zeta})+\left[N / b(\boldsymbol{\zeta})-1 / \Delta v_{N}(\boldsymbol{\zeta})\right]^{-1}$,

where $\zeta=\left(r_{s}, \zeta\right), E_{N}(\zeta)$ is the total energy of the finite periodic system, $b$ and $E_{\infty}$ are fitting parameters, and $\Delta t_{N}$ and $\Delta v_{N}$ describe the size dependence of Hartree-Fock ki- netic and exchange energies $\left(\Delta t_{N}=t_{N}^{\mathrm{HF}}-t_{\infty}^{\mathrm{HF}}, \Delta v_{N}=\right.$ $\left.v_{N}^{\mathrm{HF}}-v_{\infty}^{\mathrm{HF}}\right)$. The parameter $E_{\infty}$ is the extrapolated value of the total energy per electron. Following Ref. [5] we assume $b(\boldsymbol{\zeta})=b_{0}\left(r_{s}\right)+b_{1}\left(r_{s}\right) \zeta^{4}$. The parameters $b_{0}\left(r_{s}\right)$ and $b_{1}\left(r_{s}\right)$ are obtained from the VMC total energies of an extended set of systems $(N=1062,1450$, and 1930 for $\zeta=0 ; N=531,725$, and 965 for $\zeta=1$ ). This form is used to extrapolate all the energies computed by VMC and DMC for unpolarized and partially and fully polarized fluid systems. For lower densities the extrapolation is less critical but still important. We verified that for the crystal phases the finite-size extrapolation error is comparable to the statistical one if $N>500$. Therefore, the results for $N=686$ (bcc) and $N=864$ (fcc) are assumed to be equal to the $N \rightarrow \infty$ limit.

Extensive DMC calculations are performed for systems with $N=1062$ (paramagnetic fluid), $N=725$ (ferromagnetic fluid), $N=686$ (ferromagnetic and antiferromagnetic bcc crystal), and $N=864$ (ferromagnetic fcc phase). With the exception of $r_{s}=10, \zeta=0$, the results of the present computation agree well with those of Ref. [5]. Representative values for the correlation energy extrapolated to the thermodynamic limit are reported in Table I, and Fig. 1 shows a phase diagram displaying the stability range of the paramagnetic fluid, ferromagnetic fluid, and bcc (ferromagnetic) Wigner phases. It is apparent that a transition from the paramagnetic to a ferromagnetic fluid phase takes place around $r_{s} \approx 25$, while from the fluid to the crystal it is at $r_{s} \approx 65$. We found that the fcc is slightly less stable than the bcc structure and more stable than the fluid phase at $r_{s}=70$. The total energy difference between the two Wigner phases is 5 times smaller than the one between fluid and bcc crystal, and is, therefore, at the limit of our resolution $\left[E_{\text {tot }}(\mathrm{fcc})-E_{\mathrm{tot}}(\mathrm{bcc})=3.2 \times 10^{-4} \mathrm{eV} /\right.$ electron, to be compared with an error bar of $2.3 \times 10^{-4} \mathrm{eV} /$ electron for the total energy of either the bcc and fcc phases]. The close competition of the fluid and different crystal forms for $r_{s} \approx 70$ suggests that a metastable amorphous phase could be formed in this region of the phase diagram, because the large number of available configurations and the tiny energy differences could make the crystallization kinetics exceedingly slow.

TABLE I. Minus the DMC correlation energies (in eV) extrapolated to the thermodynamic limit. For each $\zeta$, the first row refers to the present results, while the second and third rows correspond to Ref. [5] and Ref. [4], respectively. The statistical error affects the last decimal digit.

\begin{tabular}{llcccc}
\hline \hline$r_{s}$ & 1 & 10 & 20 & 50 & 100 \\
\hline$\zeta=0$ & 1.531 & 0.497 & 0.313 & 0.1531 & \\
& 1.53 & 0.512 & & & \\
& 1.62 & 0.505 & 0.313 & 0.155 & 0.0868 \\
$\zeta=1$ & 0.814 & 0.282 & 0.1837 & 0.0968 & 0.0549 \\
& 0.794 & 0.281 & & & \\
& & 0.286 & 0.1844 & 0.0965 & 0.0564 \\
\hline \hline
\end{tabular}


We now turn to the analysis of the spin dependence of the total energy near the ferromagnetic and freezing transitions. To ease the analysis, we perform a detailed investigation at the $\mathrm{VMC}$ level and then test the validity of the resulting picture by a few DMC calculations.

Regarding the magnetic phase transition, we compute the total energy for several sizes and partial spin polarizations [14] in the range $0.8 \leq r_{s} \leq 30$. The extrapolated results for $r_{s}=25$ are reported in Fig. 2. It is clear that, at this density, the ground state has partial spin polarization (weak ferromagnet). The full set of data is used to fit an interpolation for the total energy:

$$
\begin{aligned}
E_{\mathrm{tot}}(\zeta)= & E_{\mathrm{tot}}^{\mathrm{HF}}(\zeta)+\epsilon_{0}\left(r_{s}\right) \\
& +\left[\epsilon_{1}\left(r_{s}\right)-\epsilon_{0}\left(r_{s}\right)\right] \cdot\left[\Gamma_{1}\left(r_{s}\right) \zeta^{2}+\Gamma_{2}\left(r_{s}\right) \zeta^{4}\right],
\end{aligned}
$$

where $E_{\mathrm{tot}}^{\mathrm{HF}}(\boldsymbol{\zeta})$ is the Hartree-Fock energy. In this expression $\epsilon_{0(1)}\left(r_{s}\right)$ is the correlation energy of the paramagnetic(ferromagnetic) fluid and together with $\Gamma_{1}\left(r_{s}\right)$ and $\Gamma_{2}\left(r_{s}\right)$ are fitted to the functional form

$$
\mathcal{G}\left(r_{s}\right)=-2 A\left(1+\alpha_{1} r_{s}\right) \ln \left[1+\frac{1}{2 A\left(\beta_{1} r_{s}^{1 / 2}+\beta_{2} r_{s}+\beta_{3} r_{s}^{3 / 2}+\beta_{4} r_{s}^{2}\right)}\right],
$$

whose motivation and connection to the exact highdensity limit for $\epsilon_{0(1)}$ are discussed in Ref. [15]. This expression for the energy is minimized with respect to $\zeta$ at fixed $r_{s}$ to identify the ground state spin polarization. The stable $\zeta$ as a function of $r_{s}$ is shown in the inset in Fig. 2. It is manifest that the magnetic transition is a continuous one, in contrast to what is predicted on the basis of simpler interpolations, such as the (widely used) form proposed by Perdew and Zunger [16].

We emphasize that the interpolation used to determine the ground state polarization is based on VMC calculations. Nevertheless, as mentioned above, the total energy difference between the VMC and DMC results is small and has a smooth and predictable dependence on $\zeta$. Adding $\delta E(\zeta)$ to the fitted total energies does not change the transition from continuous to discontinuous, although it moves it slightly towards higher values of $r_{s}$ (e.g., $\zeta=0.5$ polarization is stable at $r_{s}=26$ in $\mathrm{VMC}$, while it is at $r_{s}=30$ in DMC). We also remind the reader that our calculations rely on the fixed-node (FN) approxi-

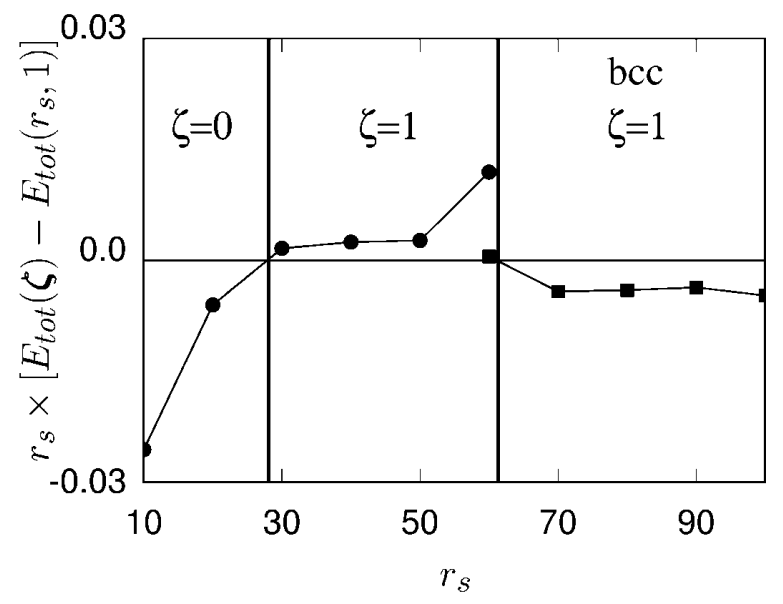

FIG. 1. Total energy difference (hartree) times $r_{s}$ of $(\bullet)$ the paramagnetic and the ferromagnetic fluids; (ם) the ferromagnetic bcc crystal and the ferromagnetic fluid. The statistical error bar is comparable to the size of the symbols. The ferromagnetic fluid is stable when both symbols are above zero. mation, and, at present, we cannot exclude that releasing the nodal constraints could change the nature of the magnetic transition. However, recent computations reported in Ref. [13] show that the FN error decreases rapidly with decreasing density and is small already at $r_{s}=20$.

Additional evidence supporting the picture of a magnetic instability and a continuous phase transition is provided by the analysis of the radial distribution function and structure factor, displaying for $r_{s} \approx 30$ long range spin-spin correlations even for nominally unpolarized systems $\left(N_{\uparrow}=N_{\downarrow}\right)$. This is illustrated in Fig. 3,

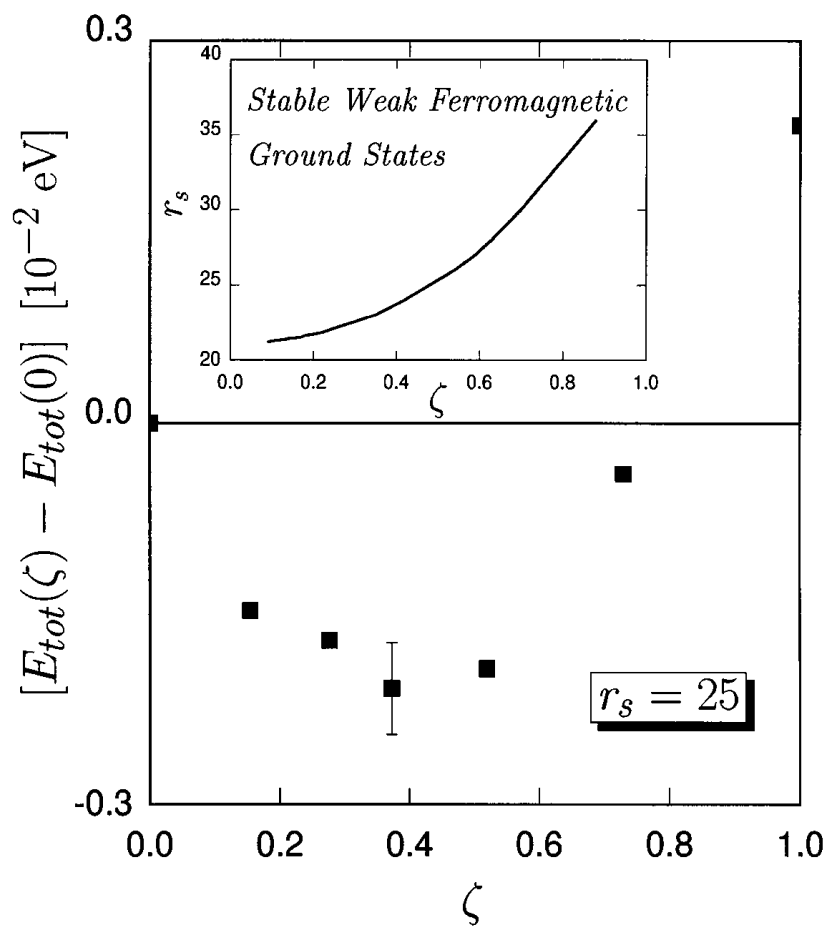

FIG. 2. Total energy (VMC) as a function of $\zeta$ for a weak ferromagnetic state. The statistical error bar is reported for the lowest energy point. The inset shows the equilibrium spin polarization $\zeta$ as a function of $r_{s}$. For $\zeta \sim 0$ and $\zeta \sim 1$ the results depend significantly on the interpolation (see text), and therefore they have not been reported in the figure. 


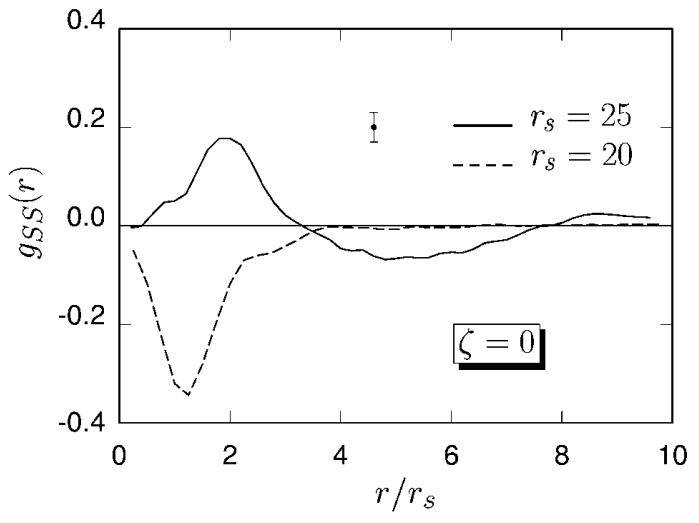

FIG. 3. Spin-spin correlation function (VMC) near the magnetic instability. A typical error bar is reported.

where the spin-spin radial distribution function $\left(g_{S S}(r)=\right.$ $\left.2\left[g_{\Uparrow}(r)-g_{\uparrow \downarrow}(r)\right]\right)$ is plotted for two densities close to the instability [17]. At $r_{s}=20, g_{S S}$ displays the expected depletion hole for parallel spins, reflecting the preference of the system for spin alternation at short distances. At $r_{s}=25$, instead, the spin correlation is small and positive at short range, and oscillating at long range, pointing to the formation of magnetic domains with partial spin polarization. This interpretation in terms of domains is confirmed by careful analysis of snapshot configurations.

Indirect support to our findings comes also from recent experiments performed in doped hexaborides $\left(\mathrm{Ca}_{1-x} \mathrm{La}_{x} \mathrm{~B}_{6}\right)$ [18]. These authors report a weak ferromagnetic phase at low carrier concentration $\left(r_{s}=28\right)$ with an ordered moment corresponding to a partial spin polarization of about $10 \%$. Moreover, the observed Curie temperature, which is as high as $600 \mathrm{~K}$, is of the same order of magnitude as the Fermi energy indicating that this represents the natural energy scale of the spin system.

The magnetic phase diagram near the Wigner phase transition is somewhat simpler. For $70 \leq r_{s} \leq 100$ the exchange energy, although small in absolute terms, is still 1 order of magnitude larger than the correlation energy, and therefore it is not surprising that the ferromagnetic bcc structure is more stable than the antiferromagnetic spin ordering. However, at both the VMC and DMC levels, the energy difference between these two states is very small $(\sim 1 \%$ of the correlation energy), and only the systematic trend $E_{\text {tot }}^{\text {ferro }}<E_{\text {tot }}^{\text {antiferro }}$ for $70 \leq r_{s} \leq 100$ gives us some confidence in this result.

In conclusion, using stochastic methods we have studied the magnetic and freezing quantum transitions of the fermionic one component plasma. We find that, contrary to the commonly accepted picture, the paramagnetic to the ferromagnetic fluid transition is a continuous one, occurring over the $20 \pm 5 \leq r_{s} \leq 40 \pm 5$ density range, which has apparently been approached in experiments by doping highly correlated solids [18]. The transition to the bcc Wigner crystal is first order, it occurs at $r_{s}=65 \pm 10$, and joins two fully polarized spin states.
Where available, our results for the transition densities disagree substantially from those of a previous study [4]. We think that the disagreement is due to the extrapolation of the finite size results to the thermodynamics limit. In our case, the importance of this extrapolation has been limited by performing computations for systems with up to 2000 electrons, in this way reducing the corresponding uncertainties. Finally, we recall that our analysis is based on a selected set of broken symmetry states. There exists the possibility of other instabilities such as the inhomogeneous spin-density-wave state or superconducting states which we have not considered in the present calculation.

G. O. thanks the Aspen Center for Physics for its hospitality. We are thankful to E. Abrahams, G. Bachelet, Z. Fisk, M. Jones, R. O. Jones, K. Sturm, and S. Trugman for illuminating discussions.

[1] E. P. Wigner and F. Seitz, Phys. Rev. 43, 804 (1933).

[2] D. Pines and P. Nozières, The Theory of Quantum Liquids (Addison-Wesley, New York, 1989), Vol. I.

[3] The continuous nature of this transition has been qualitatively anticipated by B. Alder, D. M. Ceperley, and E. L. Pollock, Int. J. Quantum Chem. Quantum Chem. Symp. 16, 49 (1982).

[4] D. M. Ceperley and B. J. Alder, Phys. Rev. Lett. 45, 566 (1980).

[5] G. Ortiz and P. Ballone, Phys. Rev. B. 50, 1391 (1994); 56, 9970 (1997).

[6] K. S. Singwi and M. P. Tosi, in Solid State Physics, edited by H. Ehrenreich, F. Seitz, and D. Turnbull (Academic, New York, 1981), Vol. 36, p. 177.

[7] At the DMC level this approximation is irrelevant because those "bosonic" correlations are fully recovered in the diffusion process; only the variance of averages measured in the simulation is affected.

[8] T. Kato, Commun. Pure Appl. Math. 10, 151 (1957).

[9] C.-J. Huang, C. Filippi, and C. J. Umrigar, J. Chem. Phys. 108, 8838 (1998).

[10] C. Umrigar, J. Wilkins, and K. Wilson, Phys. Rev. Lett. 60, 1719 (1988).

[11] P. J. Reynolds, D. M. Ceperley, B. J. Alder, and W. A. Lester, Jr., J. Chem. Phys. 77, 5593 (1982); C. J. Umrigar, M.P. Nightingale, and K. J. Runge, J. Chem. Phys. 99, 2865 (1993).

[12] We find $\delta E$ is $0.027 \mathrm{eV}$ at $r_{s}=1$ and $0.0059 \mathrm{eV}$ at $r_{s}=10$ with $\zeta=0$.

[13] Y. Kwon, D. M. Ceperley, and R. M. Martin, Phys. Rev. B 58, 6800 (1998).

[14] $N=1256, \quad \zeta=0.154 ; \quad N=1136, \quad \zeta=0.276 ; \quad N=$ $1056, \quad \zeta=0.373 ; \quad N=954, \quad \zeta=0.520 ; \quad N=838$, $\zeta=0.730$. DMC computations have been done for $r_{s}=20,25$, and $30, \zeta=0.373$ and $\zeta=0.520$.

[15] J. P. Perdew and Y. Wang, Phys. Rev. B 45, 13244 (1992).

[16] J. P. Perdew and A. Zunger, Phys. Rev. B 23, 5048 (1981).

[17] For consistency with Fig. 2, we report in Fig. 3 the results of the VMC calculation. A similar picture is obtained by DMC at a slightly lower density.

[18] D. P. Young et al., Nature (London) 397, 412 (1999). 